\title{
Overcoming the drawbacks of a revenue-sharing contract through a support program
}

\author{
Pietro De Giovanni • Maria Roselli
}

Published online: 4 April 2012

(C) The Author(s) 2012. This article is published with open access at Springerlink.com

\begin{abstract}
In a marketing channel governed by goodwill dynamics, players adjust their pricing and advertising strategies when shifting from a wholesale price contract (WPC) to a revenue sharing contract (RSC). We demonstrate that this shift is not payoff-Pareto-improving when the retailer, who is the player transferring the share of revenues, is myopic. Further, we identify the conditions under which the negative effects an RSC creates may be alleviated when the manufacturer offers a support program. Finally, contrarily to operational coordination instruments such as an RSC, a support program always leads to a payoff-Paretoimproving situation and thus should be preferred by firms to reach coordination.
\end{abstract}

Keywords Marketing channel · Contracting · Revenue-sharing contract · Support program $\cdot$ Stackelberg feedback strategies

\section{Introduction}

The seminal work by Cachon (2003) "Supply Chain Coordination with Contracts" has introduced the concept of coordination in the supply chain management literature. SC coordination refers to the implementation of a contract scheme that leads to a win-win situation and turns out to be profit-improving. Pasternak (2002), for instance, has showed the effectiveness of a rebate contract in coordinating a channel, in the sense that all players get some economic benefits under that contract regime. Cachon and Lariviere (2005) demonstrate that coordination may be achieved through a revenue sharing contract in a simple SC composed of one manufacturer and one retailer. Cachon (2003) carefully reviewed the most frequently used contract in SC coordination, highlighting strengths and weaknesses, as well

P. De Giovanni (凶)

Department of Information, Logistics and Innovation, VU University Amsterdam, de Boelelaan 1105, 3A-31, $1061 \mathrm{HV}$, Amsterdam, The Netherlands

e-mail: pietro.degiovanni@vu.nl

M. Roselli

Policlinico San Matteo di Pavia, Viale Golgi, 19, 27100, Pavia, Italy 
as benefits and drawbacks. Broadly speaking, the adoption of a contract scheme has the purpose of inducing players' strategies through increasing the economic outcomes of the entire channel rather than only maximizing their own payoff function (Pasternack 2002). SC coordination, as introduced by Pasternack (2002) and Cachon (2003), consists of adopting a contract scheme to reach a total channel profits as close as possible to the vertical integration scenario, which thus represents an upper-bound benchmark.

The marketing literature, instead, is characterized by two different research streams. On one hand, it associates the concept of coordination to vertical integration, which consists of summing up the players' value functions and optimizing with respect to only one objective function. Jørgensen and Zaccour (2003a) model channel coordination scenario as vertical integration scenarios, where the channel members agree to make coordinated decisions acting as one firm when determining its advertising and promotion strategies. On the other hand, channel coordination has been associated with providing some incentives in order to modify players' strategies. For instance, a suitable coordinating device is profit sharing, whose idea is to induce a relationship between total channel profits and individual profits such that if anyone of these is maximized, they are all maximized (Jeuland and Shugan 1983; Moorthy 1988). Other mechanisms are directly linked to players' strategies. Jørgensen et al. (2000) suggest that a support program coordinates a channel when the manufacturer designs an intertemporal retailer advertising support program that creates an incentive for the retailer to choose her advertising efforts in the way that the manufacturer desires. Jørgensen and Zaccour (2003a) build up an incentive by which the manufacturer conditions his advertising decision on the retailers' current promotion. Jørgensen and Zaccour (2003b) show how coordination can be achieved when all the channel members apply appropriate incentive strategies and those strategies are credible. Jørgensen et al. (2006) show how a manufacturer has to construct an incentive to lead a retailer to advertise at the same level of the vertical integration scenario.

Contracting in SCM follows exactly this direction of the second stream of marketing research. As Tsay et al. (1999) explain, contracts provide a means for bringing the total chain profits to the vertical integration scenario profits, achieving coordination by identifying the intra-chain dynamics that create inefficiencies, and modifying the structure of the relationships to more closely align individual incentives with global optimization. Applications of contracts are really successful in business practices: for instance, Blockbuster coordinated its channel by means of a revenue-sharing contract (Dana and Spier 2001), IBM uses a lease contract to control the reverse flows (Fleischmann et al. 2002), while Dillard's Department Stores, JCPenney, and Wal-Mart have adopted a rebate sales contract with VMI to reach coordination (Wong et al. 2009). However, contracting is not always suitable for SC and marketing channel coordination as its success may depend on several features.

Revenue sharing contract (RSC) is a coordination scheme that has attracted most of the attention of research and practice. One of its main strengths is the mitigation of the double marginalization effect when the demand depends on price because the wholesale and, consequently, retail price turns out to be lower than in a scenario without an RSC. Due to this motivation, an RSC is also called a two-parameter contract. Recent research in supply chain management has shown that an RSC works perfectly to coordinate a one-supplier, one-retailer chain in applications on the video-rental industry (Cachon and Lariviere 2005). Although research in game theory showed the benefits of an RSC in various applications (e.g., Pan et al. 2010; Cachon and Lariviere 2005; El Ouardighi et al. 2008; Geng and Mallik 2007; Dana and Spier 2001; Wang 2006; Yao et al. 2008; El Ouardighi and Kim 2010), some limitations exist. For instance, an RSC does not apply in industries in which transaction costs are really high (De Giovanni 2011b), 
when chain competition occurs (Cachon and Lariviere 2005), or when the implementation of information and auditing systems is too complex. Adopting an RSC, Blockbuster pays a wholesale price of $\$ 8$ instead of $\$ 65$ to its suppliers which get 30-45\% of the company's revenues (Cachon and Lariviere 2005). However, by adopting an RSC, Blockbuster has changed the way of managing the business into the video-rental sector. Yet, Antioco competes adequately with Blockbuster only if it transfers $55 \%$ of its revenues to its suppliers, which results in a definitely inefficient deal for the company (Kadlec et al. 1998). This paper contributes in this direction, identifying other negative aspects emerging from the adoption of an RSC in a simple marketing channel while suggesting possible countermeasures to be undertaken.

We characterize a differential game of marketing and compare players' strategies and payoffs of coordinated and non-coordinated scenarios, as carried out by means of an RSC and a wholesale price contract (WPC), respectively. We confine our interest to a singlemanufacturer, single-retailer chain. Both players invest in advertising in order to build up goodwill dynamics, the manufacturer controls the wholesale price while the retailer decides the retail price. In a coordinated scenario, the retailer transfers part of the revenues to the manufacturer to modify strategies. By contrasting strategies and payoffs in coordinated and non-coordinated scenarios, this paper addresses the following research questions:

1. How are players' strategies and payoffs influenced by adopting an RSC?

2. Is coordination by an RSC payoff-Pareto-improving?

3. What are the drawbacks an RSC implies in a channel?

In attempting to answering these research questions we faced some drawbacks of an RSC. The most important depends on the relationship between revenues shared and contribution to goodwill: when the player who transfers the share also contributes to the state, it turns out to behave myopically, focusing on static rather than dynamic tools. This implies lower profits for the entire chain. Thus, we identify the remedies to this drawback in the implementation of a support program. A well-established result is that support programs in marketing channels may be Pareto-improving under specific conditions. For example, Jørgensen et al. (2003) emphasized the benefits a manufacturer obtains when supporting a retailer's advertising expenditures. Both Karray and Zaccour (2006) and Jørgensen et al. (2000, 2001) demonstrated that a support program may exert some positive economic advantages. According to Jørgensen et al. (2003), when both advertising and promotion contribute to goodwill, a support program is beneficial only when the level of brand image is intermediate and the negative effect of promotion is not too damaging. He et al. (2009) demonstrated that offering a support program may depend on the players' profit margins: A manufacturer is willing to support the retailer's advertising efforts only when the retailer's margin is lower than the manufacturer's margin or close to it. De Giovanni (2011a) showed that a support program is payoff-Pareto-improving only when a retailer contributes substantially to the stock of goodwill and independently of the contribution of a manufacturer. We show that a support program may alleviate the drawbacks due to the adoption of an RSC. Finally, this paper provides four main contributions in the literature:

1. Contrary to the literature on contracting - and specifically on RSC and coordinationthat consists of static research (e.g., Cachon and Lariviere 2005) or dynamic research in open loop strategy (e.g., El Ouardighi et al. 2008), we characterize a Markovian feedback strategy as real business and practice update their strategies in any instant of time; therefore feedback solutions supply more valuable information than static or open loop solution. 
2. This study is the first in evaluating the effectiveness of an RSC when the player who transfers a share of revenues also does advertising to contribute to goodwill. Current research introducing the goodwill dynamic and advertising model games, in which the party who transfers part of its revenues no longer contributes also to the state (e.g., El Ouardighi et al. 2008);

3. We make the assumption that the wholesale price is a decision variable rather than a parameter. Research in static (e.g., Yao et al. 2008) and dynamic literature (e.g., El Ouardighi and Kim 2010) has considered the wholesale price exogenously. The core issue when adopting an RSC relates to how the player beneficiary of the share lowers the wholesale price. Modelling the wholesale price as a control variable provides thus a better understanding on how it decreases when an RSC is put in place.

4. While previous research used either marketing coordination mechanisms (e.g., support programs) or operational coordination scheme (e.g., contracting), this research combines the benefits of both instruments in achieving coordination.

The paper is organized as follows. Section 2 states the differential game model, while Sect. 3 characterizes the Stackelberg equilibria under non-coordinated and coordinated scenarios. Section 4 compares both strategies and outcomes, also highlighting the limitations of an RSC; thus, Sect. 5 characterizes a differential game in which an RSC is complemented with a support program. Finally, Sect. 6 reports the concluding remarks.

\section{The model}

A channel is composed of one manufacturer, player $M$, and one retailer, player $R$. Players focus on the use of marketing strategies to maximize their own payoffs. The manufacturer decides the wholesale price, $\omega(t)$ as well as advertising efforts, $A_{M}(t)$; the retailer controls both the retail price and the advertising efforts, expressed by $p(t)$ and $A_{R}(t)$, respectively. Each player's advertising efforts contribute to goodwill. In order to capture the effects of advertising in building goodwill, we used an extended version of Nerlove-Arrow's (1962) goodwill equation, which already has been used in marketing research (e.g., Jørgensen and Zaccour 2003a, 2003b) and evolves according to the following dynamics equation:

$$
\dot{G}=a A_{M}(t)+b A_{R}(t)-\delta G(t), \quad G(0)=G_{0} \geq 0,
$$

where $\delta \geq 0$ is the forgetting effect that explains the natural reduction of the stock of goodwill while $a \geq 0$ and $b \geq 0$ are the marginal contributions of the retailer's advertising efforts to this stock. We call these parameters advertising effectiveness. When these parameters assume small values, the advertising media exhibits a low effectiveness. The accumulation of this stock represents a critical point for the channel, whose demand depends on goodwill and on price as follows:

$$
D(p(t), G(t))=\theta \sqrt{G(t)}-\beta p(t)
$$

where $\beta \geq 0$ and $\theta \geq 0$ represent the effects of pricing and goodwill, respectively, on current sales. In order to satisfy the positivity assumption on demand, we need to satisfy the condition $p(t)<\frac{\theta}{\beta} \sqrt{G(t)}$.

We initially characterize two scenarios. The first is modeled by assuming an absence of coordination between players. The channel uses a WPC to manage each transaction, which implies that the manufacturer sells some products to the retailer at a wholesale price while 
the retailer sells those products at a higher retailer price, causing the double marginalization effect. Although the latter implies some inefficiencies and leads to lower demand, it is frequently used in business practices because it is simple to administer and implement; moreover, it needs simple auditing mechanisms to be put in place. In the second scenario, we model coordination using an RSC. One player — the retailer in our model - transfers part of its revenues to the other player in order to get a reduction in the wholesale price, thereby lowering the double marginalization effect. Although its theoretical assumptions are quite appealing, this contract has been demonstrated to be suitable for applications only in few industries (e.g., video-rental industry Cachon and Lariviere 2005), while the administrativerelated burdens, the auditing techniques to be adopted, as well as the information systems to be put in place represent the main barriers for its application in several industries (De Giovanni 2011b).

Contrary to previous research in operations and marketing using an RSC and assuming the wholesale price to be exogenous (e.g., Cachon and Lariviere 2005), we model it as a control variable. This way of modeling the wholesale price is consistent with the adoption of an RSC, where the player who gets a part of the other player's revenues lowers the wholesale price. $\phi \in(0,1)$ is the sharing parameter, which represents the fraction of the retailer's revenues transferred to the manufacturer that is assumed to be exogenous. We purposely disregard the case with $\phi=1$, which never happens in real business practice and leads to a vertical integration scenario. We assume that the retailer transfers a fraction of his gross revenues; as a result, adequately defining the sharing parameter becomes essential to succeed and achieve positive profits. When $\phi=0$, the players adopt a WPC. In accordance with the literature of marketing and operations using an RSC (e.g., El Ouardighi et al. 2008; Cachon and Lariviere 2005; Cachon 2003; Pan et al. 2010; Geng and Mallik 2007; Wang 2006; Yao et al. 2008; Dana and Spier 2001; De Giovanni 2011b; El Ouardighi and Kim 2010) and the research adopting a sharing profit mechanism (e.g., Chintagunta and Jain 1992; Jørgensen and Zaccour 2003a, 2003b; Jørgensen et al. 2003, 2006) we keep the assumption that the sharing parameter is exogenous; considering it as a strategy leads to an over specification of the model, where all possible elements are computed and thus the differences among scenarios vanishes. Moreover, the manufacturer decides the participation rate, which represents the percentage of retailer's advertising efforts that he wants to pay. The support strategy is represented by $B(t)$, so when no support program is offered $B(t)=0$, while when the manufacturer implements this strategy $B(t)>0$. The choices of the contract scheme and the support program also influence advertising strategies, which are investigated by means of convex functions that takes the quadratic form:

$$
C_{i}\left(A_{i}(t)\right)=\frac{A_{i}(t)^{2}}{2}
$$

where $i=M, R$. Assuming an infinite time horizon and a positive discount rate $\rho$, the manufacturer's objective functional under an RSC complemented with a support program is:

$$
J_{M}=\int_{0}^{\infty} e^{-\rho t}\left\{[\theta \sqrt{G(t)}-\beta p(t)][\phi p(t)+\omega(t)]-\frac{A_{M}(t)^{2}}{2}-\frac{B(t) A_{R}(t)^{2}}{2}\right\} d t
$$

and the retailer's objective functional is:

$$
J_{R}=\int_{0}^{\infty} e^{-\rho t}\left\{[\theta \sqrt{G(t)}-\beta p(t)][p(t)(1-\phi)-\omega(t)]-\frac{(1-B(t)) A_{R}(t)^{2}}{2}\right\} d t
$$


Using Eqs. (1), (4), and (5), we have defined a two-player differential game with five controls, $A_{i}(t) \geq 0, \omega(t) \geq 0, p(t) \geq 0$, and $B(t) \geq 0$, and one state variable, $G(t) \geq 0$. From now on, the time argument is omitted to avoid confusion.

\section{Scenarios}

We characterize and compare three equilibrium solutions and confine our interest to stationary strategies, that is, strategies which depend on the state only (i.e., not on time). Restricting one's interest to stationary strategies is a standard working hypothesis in autonomous dynamic optimization problems with infinite time horizon. Strategies are evaluated at the steady-state; they are current-state-dependent as well as time-independent.

Benchmark (non-coordinated) scenario Each player decides his strategies to maximize his own payoff independently of the other player's strategies. The retailer does not share his revenues with the manufacturer, thus, $\phi=0$, while the manufacturer does not support the retailer's advertising efforts, thus $B=0$. This scenario is played in a feedback Stackelberg game in which the manufacturer is the leader. We use the subscript " $N$ " to signify a "noncoordinated scenario."

Coordinated scenario The retailer transfers a fraction of her revenues to the manufacturer to obtain a reduction of the wholesale price, thereby alleviating the double marginalization effect. The new manufacturer's strategies are computed also considering the sharing parameter, $\phi \in(0,1)$. Even though, the manufacturer does not participate in the retailer's advertising efforts, therefore $B=0$. Players adjust their pricing and advertising strategies according to this combination of contract and support program, and we sought a feedback Stackelberg game in which the manufacturer is the leader. We use the subscript " $C$ " to signify the implementation of a "coordinated" scenario.

Dual coordination mechanisms scenario An RSC is complemented with a support program, therefore $\phi \in(0,1)$ and $B \in(0,1)$. The retailer transfers a percentage of her revenues to the manufacturer while the manufacturer supports the retailer's advertising investments. We exclude the case when $B=1$ because it will lead to a vertical integrated scenario (see note 1). Also in this case, we look for a feedback Stackelberg game in which the manufacturer is the leader. We use the subscript " $D$ " for referring to the "dual coordination mechanisms" scenario.

Note that we disregard the case in which a WPC is complemented with a coop program since the results are aligned with the existing literature (e.g., Jørgensen et al. 2001). Moreover, we conjecture linear value functions for each scenario knowing that:

1. Long term strategies are also influenced by the "dynamic" parameters $a, b, \delta$ (goodwill dynamics) and $\rho$ (discount rate) (e.g., Jørgensen et al. 2000), which never appear in static formulations;

2. Advertising strategies imply a carry over effect that can be captured only when introducing a stock variable representing brand goodwill (e.g., Eq. (1)) (see e.g., Chintagunta and Jain 1992; Jørgensen and Zaccour 2005); and

3. Modeling the carry over effect of advertising by the goodwill dynamics even when conjecturing linear value functions is actually common in differential games research in order to derive explicit solutions (e.g., Jørgensen et al. 2000; Jørgensen and Zaccour 2003a, 2003b). 


\section{Equilibria}

We derive the equilibria in the coordinated and benchmark scenarios in the following subsections and further comparing strategies and outcomes. This comparison allows for identifying the limitations of an RSC under our model assumptions.

\subsection{Equilibria in a non-coordinated scenario}

Under a WPC scenario, each player aims to maximize his own payoff independently of the other player's strategies. Both players seek to contribute to building up goodwill, which enhances the demand and positively influences both players' payoffs. On the other hand, the demand negatively depends on price; thus, both pricing and advertising play a key role for both players. Although a WPC still involves the problem of the double marginalization effect, it is the simplest contract scheme to be adopted in business practice. It is intuitive and based on simple price transaction; it requires neither specific auditing systems nor particular information systems to be put in place, while the administrative burdens the players face are marginal. Most research in marketing uses this contract to manage transactions among players, while only recently has research in contracting proposed alternative and more sophisticated contracts (e.g., Cachon 2003) that seem to be contest-specific rather than generalizable. In this sense, channels and supply chains obtain successful economic benefits even when using a WPC, although it presents several inefficiencies (e.g., the double marginalization effect). All players throughout the channel add a markup, causing the final price to be higher and the final demand to be lower than would be the case if firms were vertically integrated (Tirole 1988). Nonetheless, it is quite diffused in managing players' transactions. We characterize the equilibrium strategies in the following proposition.

Proposition 1 The equilibrium wholesale price, retail price, and advertising strategies in a non-coordinated scenario are given by:

$$
\begin{aligned}
\omega^{N} & =\frac{\theta \sqrt{G^{N}}}{2 \beta}, \\
p^{N} & =\frac{3 \theta \sqrt{G^{N}}}{4 \beta}, \\
A_{M}^{N} & =\frac{a \theta^{2}}{8 \beta(\rho+\delta)}, \\
A_{R}^{N} & =\frac{b \theta^{2}}{16 \beta(\rho+\delta)}
\end{aligned}
$$

and the value functions assume the following forms:

$$
\begin{aligned}
& V_{M}^{N}=\frac{\theta^{2}}{8 \beta(\rho+\delta)} G^{N}+\frac{\varphi_{1}^{N}\left(a^{2} \varphi_{1}^{N}+2 b^{2} \varsigma_{1}^{N}\right)}{2 \rho}, \\
& V_{R}^{N}=\frac{\theta^{2}}{16 \beta(\rho+\delta)} G^{N}+\frac{\varsigma_{1}^{N}\left(2 a^{2} \varphi_{1}^{N}+b^{2} \varsigma_{1}^{N}\right)}{2 \rho}
\end{aligned}
$$

Proof See Appendix. 
Both wholesale price and retailer price are state-dependent and for both it turns out that $\frac{\partial \omega^{N}}{\partial G^{N}}=\frac{\theta}{4 \beta \sqrt{G^{N}}} \geq 0$ and $\frac{\partial p^{N}}{\partial G^{N}}=\frac{3 \theta}{8 \beta \sqrt{G^{N}}} \geq 0$. This result is in line with Taboubi and Zaccour (2002), who demonstrated a positive relationship between pricing strategies and goodwill. In contrast, advertising strategies are constant (i.e., state-independent). This result depends on the missing interfaces between goodwill and advertising efforts in (4) and (5). Nonetheless, the players' value functions are state-dependent, resulting in the following:

$$
\begin{aligned}
& \frac{\partial V_{M}^{N}}{\partial G^{N}}=\frac{\theta^{2}}{8 \beta(\rho+\delta)} \geq 0, \\
& \frac{\partial V_{R}^{N}}{\partial G^{N}}=\frac{\theta^{2}}{16 \beta(\rho+\delta)} \geq 0
\end{aligned}
$$

Such evidence motivates managers and practitioners to build up their stock of goodwill as high as possible and then to price more. Although advertising is state independent, (12) and (13) suggest that increasing the value of the state will benefit both players. By substituting (8) and (9) into (1), we derive goodwill at the steady-state as follows:

$$
G_{\infty}^{N}=\frac{\left(2 a^{2}+b^{2}\right) \theta^{2}}{16 \delta \beta(\rho+\delta)} \geq 0
$$

At the steady state, goodwill depends positively (negatively) on the customers' goodwill (price) sensitivity, while the large forgetting effect and discount factor values lower that stock. Notice that the contribution of the manufacturer's advertising strategy on goodwill at the steady state is twice as large as the retailer's. This result depends on the information structure of the game.

\subsection{Equilibria in a coordinated scenario}

The channel may reach coordination through the adoption of a RSC, in the sense that it is payoff-Pareto-improving. The operations literature has extensively highlighted the advantages that a RSC creates, which are mainly related to the mitigation of the double marginalization effect. Even though, we show here the implied limitations when the player who transfers the share does also advertising. As we have previously introduced, the sharing parameter is assumed to be exogenous to avoid an over specification of the model. Moreover, the structure of the game does not allow for considering the sharing parameter as a control variable. Since the retailer is the follower, when computing the sharing parameter it turns out to be null. Since the retailer influences the demand by controlling the price, she already decides an optimal price which is independent of the sharing parameter value. We follow the assumption of previous research in marketing and operations (e.g., El Ouardighi et al. 2008; Cachon and Lariviere 2005; Cachon 2003; Pan et al. 2010; Geng and Mallik 2007; Wang 2006; Yao et al. 2008; Dana and Spier 2001; De Giovanni 2011b; El Ouardighi and Kim 2010) as well as the contributions using a sharing profits mechanism (e.g., Chintagunta and Jain 1992; Jørgensen and Zaccour 2003a, 2003b; Jørgensen et al. 2003, 2006) thus considering the sharing parameter as exogenous while identifying under which values it allows a two-parameter contract to be payoff-Pareto-improving.

Proposition 2 The equilibrium wholesale price, retail price, and advertising strategies in a coordinated scenario are given by:

$$
\omega^{C}=\frac{\theta \sqrt{G^{C}}(1-\phi)^{2}}{\beta(2-\phi)},
$$




$$
\begin{aligned}
p^{C} & =\frac{\theta \sqrt{G^{C}}(3-2 \phi)}{2 \beta(2-\phi)}, \\
A_{M}^{C} & =\frac{a \theta^{2}}{4 \beta(2-\phi)(\rho+\delta)}, \\
A_{R}^{C} & =\frac{b \theta^{2}(1-\phi)}{4 \beta(2-\phi)^{2}(\rho+\delta)}
\end{aligned}
$$

and the value functions assume the following forms:

$$
\begin{aligned}
& V_{M}^{C}=\frac{\theta^{2}}{4 \beta(2-\phi)(\rho+\delta)} G^{C}+\frac{\varphi_{1}^{C}\left(a^{2} \varphi_{1}^{C}+2 b^{2} \varsigma_{1}^{C}\right)}{2 \rho}, \\
& V_{R}^{C}=\frac{\theta^{2}(1-\phi)}{4 \beta(2-\phi)^{2}(\rho+\delta)} G^{C}+\frac{\varsigma_{1}^{C}\left(2 a^{2} \varphi_{1}^{C}+b^{2} \varsigma_{1}^{C}\right)}{2 \rho}
\end{aligned}
$$

Proof See Appendix.

Changes in strategies and value functions with respect to the state follow the same line of the previous findings in a non-coordinated scenario. Although the wholesale price and retail pricing strategies increase the goodwill, advertising strategies are state-independent in the coordinated setting as well. Moreover, both value functions increase in the state; as such, both players decide their strategies in order to build up a large stock of goodwill and obtain a higher payoff. The goodwill at the steady state is derived by substituting (17) and (18) into (1), thereby resulting in the following:

$$
G_{\infty}^{C}=\frac{\theta^{2}\left[a^{2}(2-\phi)+b^{2}(1-\phi)\right]}{4 \beta \delta(2-\phi)^{2}(\rho+\delta)}
$$

The sharing parameter, $\phi$, influences the choice of implementing a RSC in a marketing channel. Both pricing and advertising strategies are consequently influenced either positively or negatively, which in turns leads to higher or lower goodwill and value functions depending on the amplitude of the model parameter values.

Corollary 1 Strategies and sharing parameter are linked by the following relationships:

(1)

$$
\frac{\partial \omega^{C}}{\partial \phi}=-\frac{\theta \sqrt{G^{C}}\left(3-4 \phi+\phi^{2}\right)}{\beta(2-\phi)^{2}} \leq 0 \quad \forall \phi \in(0,1) ;
$$

(2)

$$
\frac{\partial p^{C}}{\partial \phi}=-\frac{\theta \sqrt{G^{C}}}{2 \beta(2-\phi)^{2}} \leq 0 \quad \forall \phi \in(0,1) ;
$$

(3)

$$
\frac{\partial A_{M}^{C}}{\partial \phi}=\frac{a \theta^{2}}{4 \beta(2-\phi)^{2}(\rho+\delta)} \geq 0 \quad \forall \phi \in(0,1)
$$

(4)

$$
\frac{\partial A_{R}^{C}}{\partial \phi}=-\frac{b \theta^{2} \phi}{4 \beta(2-\phi)^{3}(\rho+\delta)} \leq 0 \quad \forall \phi \in(0,1)
$$


As expected, results (1) and (2) highlight that both the wholesale price and the retail price in a RSC decrease in the sharing parameter. This result is in the spirit of coordination by a RSC, which allows for a reduction in the double marginalization effect and an increase in sales as the demand depends on pricing (Cachon and Lariviere 2005; De Giovanni 2011b). Finally, independently of the information structure, a RSC always remains effective for reducing the double marginalization effect. Previous research has provided evidence on the effectiveness of a RSC when playing the Nash game, while we show that playing the Stalkerberg game will not corroborate its usefulness. The result in (3) supplies information on the manufacturer's willingness to do more when implementing a RSC. He increases advertising according to the sharing parameter, thereby providing further contributions to enhance the stock of goodwill. This also represents a way to push the retailer to share more, which increases advantages in terms of pricing and goodwill. The manufacturer advertises more because the share represents an economic incentive to put additional efforts into advertising. The result in (4) highlights the lower advertising efforts the retailer spends when adopting a RSC. He possesses a lower amount of economic resources while expecting the manufacturer to advertise more when receiving his share of the revenues.

The results of this corollary highlight the need to investigate the stock of goodwill at the steady state when adopting a RSC. The different changes in players' advertising strategies may lead to a lower level of goodwill when the contribution of the retailer's advertising to that stock is considerably high. Any increment of the sharing parameter increases the goodwill at the steady state when $a>b$. The derivative $\frac{\partial G_{\infty}^{C}}{\partial \phi}=\frac{\theta^{2}\left[a^{2}(2-\phi)-b^{2} \phi\right]}{4 \beta \delta(2-\phi)^{3}(\rho+\delta)}$ turns out to be positive (negative) when the term in the square brackets is positive (negative). Consequently, when there is a large difference between $a$ and $b$, such that $a \geq b \sqrt{\frac{\phi}{2-\phi}}, \frac{\partial G_{\infty}^{C}}{\partial \phi} \geq 0$; implementing a RSC contributes positively to goodwill at the steady state only when $b \in\left[0, a \sqrt{\frac{2-\phi}{\phi}}\right)$.

Therefore, implementing a RSC does not necessarily lead to higher goodwill at the steady state. Consequently, the adoption of this contract positively influences the static tools influencing the demand (e.g., price) but may negatively impact the dynamic tools (e.g., goodwill). What remains to be verified is under which conditions a RSC should be avoided because it sponsors static tools while diminishing the importance of dynamic elements. Therefore, in the next section, we compare the strategies, state, and players' payoff changes when moving from a non-coordinated to a coordinated marketing channel.

\subsection{Comparison of equilibria}

This section introduces the difference in strategies, state variable, and players' payoffs when shifting from a non-coordinated to a coordinated setting. Although Cachon and Lariviere (2005) as well as El Ouardighi et al. (2008) have shown the advantages of implementing a RSC, we highlight the drawbacks it generates in a marketing channel since the manufacturer's and retailer's advertising strategies are not aligned.

Proposition 3 The manufacturer advertises more under a coordinated scenario with a RSC since it results that $A_{M}^{C}-A_{M}^{N}>0, \forall \phi>0$.

Proof Computing the difference $A_{M}^{C}-A_{M}^{N}$, we get $A_{M}^{C}-A_{M}^{N}=\frac{a \theta^{2} \phi}{8 \beta(\delta+\rho)(2-\phi)} \geq 0$.

As the manufacturer receives a share from the retailer-which could be assimilated into an economic incentive to engage in more coordination-he advertises more for any positive 
value of the sharing parameter. When adopting a RSC, the manufacturer has two motivations to contribute to the state: the first derives from the increasing demand and the second comes from the retailer's share.

Proposition 4 The retailer advertises less under a coordinated scenario with a RSC since it results that $A_{R}^{C}-A_{R}^{N}<0, \forall \phi>0$.

Proof Computing the difference $A_{R}^{C}-A_{R}^{N}$, we get $A_{R}^{C}-A_{R}^{N}=-\frac{b \theta^{2} \phi^{2}}{16 \beta(\delta+\rho)(2-\phi)^{2}} \leq 0$.

When implementing a RSC, the retailer transfers his economic resources to the manufacturer with the purpose of inducing him to advertise more. With this reduction, the retailer contributes only marginally to the state, so that a RSC does not guarantee higher goodwill than a one-sided payment contract. The retailer has, in fact, few economic resources to devote to building up the goodwill, yet he expects the manufacturer to advertise more as a result of receiving additional economic benefits through the share. The goodwill at the steady-state turns out to be higher under coordination with a RSC when $a>b$.

The difference $G^{C}-G^{N}=\frac{\theta^{2} \phi\left[2 a^{2}(2-\phi)-b^{2} \phi\right]}{16 \beta \delta(\delta+\rho)(2-\phi)^{2}}$ turns out to be negative when $b>a \sqrt{\frac{2(2-\phi)}{\phi}}$. When this inequality holds, implementing a RSC results in a lower stock of goodwill at the steady-state.

$$
b=a \sqrt{\frac{2(2-\phi)}{\phi}}
$$

is the threshold that determines whether implementing a RSC leads to a higher goodwill at the steady-state. That stock depends not only on the contract scheme adopted, but also on the different media advertising effectiveness. When a WPC is adopted, both players advertise to increase goodwill. When a RSC is implemented, the value of the state over infinity varies according to the marginal contributions of both advertising strategies. Consider for instance the special case when $a$ is really small, which simply implies that the manufacturer has chosen a lightly effective advertising media, the adoption of a RSC destroys the value of goodwill at the steady state because the contribution of the retailer is simply more important, while his advertising efforts are lower under coordination. This is a drawback of coordination through a RSC, which lowers the importance of goodwill and then the relevance of $d y$ namic tools when the player who transfers the share of revenues also contributes to goodwill through advertising. In fact, under coordination, the retailer focuses on the nominal benefits a RSC provides (i.e., the reduction of the double marginalization effect). As a result, both the wholesale and the retail prices are lower under a coordinated scenario, indicating that a RSC promotes more static than dynamic tools. In this sense, the retailer becomes myopic when a RSC is put in place.

Proposition 5 The manufacturer fixes a higher wholesale price under a RSC when the stock accumulated under coordination is sufficiently larger than the goodwill accumulated under non-coordination.

Proof The sign of the difference $\omega^{C}-\omega^{N}=\frac{\theta\left[2 \sqrt{G^{C}}(1-\phi)^{2}-\sqrt{G^{N}}(2-\phi)\right]}{2 \beta(2-\phi)}$ depends on the difference between the stock of goodwill in coordinated and non-coordinated scenarios. When $\frac{G^{C}}{G^{N}}>\left(\frac{2-\phi}{2(1-\phi)^{2}}\right)^{2}, \omega^{C}>\omega^{N}$. 
The RSC mitigates the double marginalization effect only when the manufacturer's advertising strategy effectiveness is higher than the retailer's. In fact, in this case, the lower advertising the retailer invests no longer lowers the level of the state as well; thus, coordination through a RSC exerts the purpose of reducing the wholesale price in each transaction. In contrast, the stock of goodwill at the steady-state turns out to be very small because the manufacturer's advertising effectiveness is lower than the retailer's.

Proposition 6 The manufacturer fixes a higher retailer price under a RSC when the stock accumulated under coordination is sufficiently larger than the goodwill accumulated under non-coordination.

Proof The sign of the difference $p^{C}-p^{N}=\frac{\theta\left[2 \sqrt{G^{C}}(3-2 \phi)-3 \sqrt{G^{N}}(2-\phi)\right]}{4 \beta(2-\phi)}$ depends on the difference between the goodwill accumulated under coordinated and non-coordinated scenarios. Specifically, $p^{C}>p^{N} \forall \phi>0$ when $\frac{G^{C}}{G^{N}}>\left(\frac{3(2-\phi)}{2(3-2 \phi)}\right)^{2}$.

Ultimately, a RSC implemented to coordinate a marketing channel no longer exerts its benefits of reducing the double marginalization effect and, consequently, increasing demand when the stock of goodwill under coordination is not sufficiently larger that the non-coordinated ones. Since the retailer advertises less under a RSC, coordination by a two-parameter contract destroys the economic value when the manufacturer's advertising effectiveness is lower than the retailer's. By substituting the steady-state values of goodwill into the value functions and comparing the players' payoffs under the coordinated and non-coordinated scenarios we formulate the following proposition.

Proposition 7 The sign of the difference

$$
V_{M}^{C}-V_{M}^{N}=\frac{\theta^{4} \phi\left[a^{2}(\delta+2 \rho)\left(8-6 \phi+\phi^{2}\right)-b^{2}(\delta+\rho)\left(6 \phi-4-\phi^{2}\right)\right]}{128 \beta^{2} \delta \rho(\delta+\rho)(2-\phi)^{3}}
$$

turns out to be as follows:

(a) When goodwill does not depend on the manufacturer's advertising strategy, $V_{M}^{C}-V_{M}^{N}$ $\geq 0 \forall \phi \in(0,3-\sqrt{5})$

(b) When goodwill does not depend on the retailer's advertising strategy, $V_{M}^{C}-V_{M}^{N}$ $\geq 0 \forall \phi \in(0,1)$

(c) When goodwill depends on both players' advertising strategies, $V_{M}^{C}-V_{M}^{N} \geq 0 \forall \phi \in$ $(0,3-\sqrt{5})$ and $\forall \phi \in(3-\sqrt{5}, 1) \Leftrightarrow a>b$.

Proof In Case (a), when $a$ is sufficiently small, the polynomial $\left(4-6 \phi+\phi^{2}\right)$ gives solutions $\phi=3 \pm \sqrt{5}$, thus that difference remains positive when $\phi \in(0,3-\sqrt{5})$. In case (b), when $b$ is sufficiently small, the roots of the polynomial $\left(8-6 \phi+\phi^{2}\right)$ are both larger than 1 ; thus that difference is positive for any value of $\phi \in(0,1)$. In Case (c), that difference is positive either for any value of $\phi \in(0,3-\sqrt{5})$ or for any value of $\phi \in(3-\sqrt{5}, 1)$ whenever $a>b$, e.g., $a=\frac{1}{b} \sqrt{\frac{(\delta+2 \rho)\left(8-6 \phi+\phi^{2}\right)}{(\delta+\rho)\left(6 \phi-4-\phi^{2}\right)}}$; finally, when $\phi=3-\sqrt{5}, V_{M}^{C}=V_{M}^{N}$.

Proposition 8 The sign of the difference

$$
V_{R}^{C}-V_{R}^{N}=\frac{\theta^{4} \phi\left[4 a^{2}(\delta+\rho)\left(8-16 \phi+8 \phi^{2}-\phi^{3}\right)-b^{2}(\delta+2 \rho)\left(8-8 \phi+\phi^{2}\right)\right]}{512 \beta^{2} \delta \rho(\delta+\rho)(2-\phi)^{4}}
$$

turns out to be as follows: 
(a) When goodwill does not depend on the manufacturer's advertising strategy, $V_{R}^{C}-V_{R}^{N}$ $<0 \forall \phi \in(0,1)$;

(b) When goodwill does not depend on the retailer's advertising strategy, $V_{R}^{C}-V_{R}^{N}$ $>0 \forall \phi \in(0,3-\sqrt{5})$;

(c) When goodwill depends on both players' advertising strategies, $V_{R}^{C}-V_{R}^{N}$ $>0 \forall \phi \in(0,3-\sqrt{5}) \Leftrightarrow a>b$.

Proof In Case (a), when $a$ is sufficiently small, the polynomial $8-8 \phi+\phi^{2}>0 \forall \phi \in(0,1)$, thus $V_{R}^{C}-V_{R}^{N}<0 \forall \phi \in(0,1)$; in Case (b), when $b$ is sufficiently small, the polynomial $8-16 \phi+8 \phi^{2}-\phi^{3}>0 \forall \phi \in(0,3-\sqrt{5})$, thus $V_{R}^{C}-V_{R}^{N}>0 \forall \phi \in(0,3-\sqrt{5})$; in Case (3),

$$
V_{R}^{C}-V_{R}^{N}>0 \quad \forall \phi \in(0,3-\sqrt{5}) \Longleftrightarrow a>b,
$$

e.g.,

$$
a>\frac{b}{2} \sqrt{\frac{(\delta+2 \rho)\left(8-8 \phi+\phi^{2}\right)}{(\delta+\rho)\left(8-16 \phi+8 \phi^{2}-\phi^{3}\right)}} .
$$

Finally, whenever $\phi=3-\sqrt{5}, V_{R}^{C}=V_{R}^{N}$.

In order for a RSC to be payoff-Pareto-improving, the manufacturer's advertising medium effectiveness must be higher than the retailer's one, while the sharing parameter should not be too high [e.g., $\phi \in(0,3-\sqrt{5})]$. The manufacturer prefers the adoption of a RSC when the sharing parameter is high and the retailer's advertising effectiveness is low, while the retailer prefers the adoption of a RSC when the sharing parameter and the retailer's advertising effectiveness are both sufficiently low.

Corollary 2 When the players use the same advertising media $(a=b)$ it results that $V_{M}^{C}-$ $V_{M}^{N}>0, \forall \phi(0,1)$ and $V_{R}^{C}-V_{R}^{N}>0, \forall \phi(0,0.6377)$. Thus, the implementation of a $R S C$ makes the manufacturer always better off, while the retailer's economic advantages depend on the sharing parameter values: when it is sufficiently low, the adoption of a RSC also suits the retailer.

\section{Overcoming the drawbacks of a RSC through a support program}

In this section we characterize a special case of the game to provide additional insights on how to overcome the drawbacks of a RSC under the model assumptions. The retailer's " $m y$ opia" induced when adopting a RSC could be lessened when the manufacturer offers a support program. As it has been already shown in marketing channel literature (e.g., Jørgensen and Zaccour 2003a, 2003b) as well as in operations management (De Giovanni 2011a), a support program is generally payoff-Pareto-improving because it implies players to invest more (e.g., in advertising in order to increase the goodwill). By offering a support program, a manufacturer promotes the importance of dynamic tools at the eyes of a retailer, who focuses instead more on static elements under a RSC contract. The support program assumes the form of a participation rate, so that the manufacturer-acting as the leader-announces he will adopt support programs for advertising strategies, the retailer decides her advertising efforts, and then the manufacturer decides his participation rate. According to this sequence of events, the next proposition characterizes the equilibrium strategies under this scenario. 
Proposition 9 The equilibrium wholesale price, retailer price, advertising efforts, as well as the support strategies when complementing a RSC with a support program are characterized as follows:

$$
\begin{aligned}
& \omega^{D}=\frac{\theta \sqrt{G^{D}}(1-\phi)^{2}}{\beta(2-\phi)}, \\
& p^{D}=\frac{\theta \sqrt{G^{D}}(3-2 \phi)}{2 \beta(2-\phi)}, \\
& A_{M}^{D}=\frac{a \theta^{2}}{8 \beta(2-\phi)(\rho+\delta)}, \\
& A_{R}^{D}=\frac{b(5-3 \phi) \theta^{2}}{8 \beta(2-\phi)^{2}(\rho+\delta)}, \\
& B=\frac{3-\phi}{5-3 \phi}
\end{aligned}
$$

while the value functions turn out to be:

$$
\begin{aligned}
& V_{M}^{D}=\frac{\theta^{2}}{4 \beta(2-\phi)(\rho+\delta)} G^{D}+\frac{b^{2} \varsigma_{1}^{D}\left(2 \varphi_{1}^{D}+\varsigma_{1}^{D}\right)+2 \varphi_{1}^{D 2}\left(a^{2}+b^{2}\right)}{4 \rho}, \\
& V_{R}^{D}=\frac{\theta^{2}(1-\phi)}{4 \beta(2-\phi)^{2}(\rho+\delta)} G^{D}+\frac{\varsigma_{1}^{D}\left(4 a^{2} \varphi_{1}^{D}+b^{2}\left(2 \varphi_{1}^{D}+\varsigma_{1}^{D}\right)\right)}{4 \rho}
\end{aligned}
$$

Proof See Appendix.

As in the previous two scenarios, wholesale and retail pricing strategies depend on the state while advertising strategies are state-independent. Those strategies vary according to the sharing parameter as reported in Corollary 1. The participation strategy is stateindependent and varies exclusively according to the sharing parameter. ${ }^{1}$ As the support strategy increases in the sharing parameter (e.g., $\frac{\partial B}{\partial \phi}=\frac{4}{(5-3 \phi)^{2}}>0$ ), the manufacturer is willing to support when a RSC is implemented because he wishes to align the retailer' focus on dynamic tools. While the retailer becomes myopic under a RSC regime, a participation strategy for supporting her advertising efforts shifts her focus on dynamic tools. This result is clearly displayed in the next proposition.

Proposition 10 The retailer advertises more under a RSC with a support program than in the benchmark and coordination case, since it results that $A_{R}^{D}-A_{R}^{N}>0$ and $A_{R}^{D}-A_{R}^{C}>$ $0 \forall \phi \in(0,1)$.

Proof Computing the difference $A_{R}^{D}-A_{R}^{N}$, we get that: $A_{R}^{D}-A_{R}^{N}=\frac{b \theta^{2}\left(6-2 \phi-\phi^{2}\right)}{16 \beta(\delta+\rho)(2-\phi)^{2}}>0$ $\forall \phi \in(0,1)$. Similarly, computing the difference $A_{R}^{D}-A_{R}^{C}$, we get that: $A_{R}^{D}-A_{R}^{C}=$ $\frac{b \theta^{2}(3-\phi)}{8 \beta(\delta+\rho)(2-\phi)^{2}}>0 \forall \phi \in(0,1)$.

\footnotetext{
${ }^{1}$ Since the support strategy only depends on the sharing parameter, a full support $(B=1)$ is possible only when $\phi=1$, which finally leads to a centralized scenario. The latter is out of our interest because results in centralized scenario when all players contribute to goodwill already appeared in the literature (e.g., Jørgensen and Zaccour 2003a).
} 
Offering a support program leads to higher retailer's advertising efforts independently of the sharing parameter values. When $\phi>0$, the retailer efforts under a RSC with a support program are higher than the benchmark case. On the other hand, the manufacturer always advertises at the same level, independently of the participation rate with respect to the coordinated case; therefore, $A_{M}^{D}=A_{M}^{C}>A_{M}^{N}$. This result is in line with the literature of marketing and operations, which highlights the characteristics of a support program that induces players to do more.

Proposition 11 Goodwill stocks at the steady-state compare as follows: $G^{D}>G^{C}$ and $G^{D}>G^{N}$.

Proof Simply compute the differences between those stocks of goodwill to show that

$$
\begin{aligned}
G^{D}-G^{C} & =\frac{b^{2} \theta^{2}(3-\phi)}{8 \beta \delta(\delta+\rho)(2-\phi)^{2}}>0 \quad \forall \phi(0,1) \quad \text { and } \\
G^{D}-G^{N} & =\frac{\theta^{2}\left[2 a^{2}(3-\phi) \phi+b^{2}\left(6-2 \phi-\phi^{2}\right)\right]}{16 \beta \delta(\delta+\rho)(2-\phi)^{2}}>0 \quad \forall \phi \in(0,1) .
\end{aligned}
$$

When a RSC is complemented by a support program, the stock of goodwill always turns out to be higher than in the other scenarios while a support program alone assures higher levels of the state only under some particular conditions. Using a dual instrument mechanism leads to higher wholesale price and retailer price since they are state dependent while the other parameters no longer change.

Proposition 12 Under a RSC complemented with a support program it turns out that $V_{M}^{D}>$ $V_{M}^{C}$ and $V_{R}^{D}>V_{R}^{C}$.

Proof Compute the difference $V_{M}^{D}-V_{M}^{C}$ to show that

$$
V_{M}^{D}-V_{M}^{C}=\frac{b^{2} \theta^{4}\left[2 \rho\left(6-5 \phi+\phi^{2}\right)+\delta\left(5-4 \phi+\phi^{2}\right)\right]}{64 \beta^{2} \delta \rho(\delta+\rho)^{2}(2-\phi)^{4}}>0 \quad \forall \phi(0,1) .
$$

Further, compute $V_{R}^{D}-V_{R}^{C}$ to show that

$$
V_{R}^{D}-V_{R}^{C}=\frac{b^{2} \theta^{4}(\delta+2 \rho)\left(3-4 \phi+\phi^{2}\right)}{64 \beta^{2} \delta \rho(\delta+\rho)^{2}(2-\phi)^{4}}>0 \quad \forall \phi \in(0,1) .
$$

Corollary 3 The previous results also hold when the players use the same advertising media $(a=b)$.

Notice that the result of the previous proposition also holds when $\phi=0$, thus a support program leads to higher players' payoff independently of the contract scheme adopted. Consequently, in the particular case when $\phi=0, V_{i \mid \phi=0}^{D}>V_{i \mid \phi=0}^{C}=V_{i}^{N}$, therefore the implementation of a support program always leads to a payoff-Pareto-improving situation when $\mathrm{RSC}$ is no longer adopted. In contrast, putting in place a RSC generates some inefficiency that a support program rarely overcomes.

Proposition 13 Under a RSC complemented by a support program it always results $V_{M}^{D}>$ $V_{M}^{N}$ while it turns out that $V_{R}^{D}>V_{R}^{N}$ only when $\phi \in(0,3-\sqrt{5})$. 
Proof Compute the difference $V_{M}^{D}-V_{M}^{N}$, to show that

$$
\begin{aligned}
V_{M}^{D}-V_{M}^{N}= & \frac{\theta^{4}\left(a^{2}(\delta+2 \rho)(4-\phi)(2-\phi)^{2} \phi\right)}{128 \beta^{2} \delta \rho(\delta+\rho)^{2}(2-\phi)^{4}} \\
& +\frac{b^{2}\left[\rho\left(24-12 \phi-12 \phi^{2}+8 \phi^{3}-\phi^{4}\right)+\delta\left(10-14 \phi^{2}+8 \phi^{3}-\phi^{4}\right)\right]}{128 \beta^{2} \delta \rho(\delta+\rho)^{2}(2-\phi)^{4}} \\
> & 0 \quad \forall \phi(0,1) ;
\end{aligned}
$$

further computing the difference

$$
V_{R}^{D}-V_{R}^{N}=\frac{\theta^{4}\left(4 a^{2}(\delta+\rho) \phi\left(8-16 \phi+8 \phi^{2}-\phi^{3}\right)+b^{2}(\delta+2 \rho)\left(24-32 \phi+8 \phi^{3}-\phi^{4}\right)\right)}{512 \beta^{2} \delta \rho(\delta+\rho)^{2}(2-\phi)^{4}}
$$

we can show demonstrate that:

(a) When goodwill does not depend on the manufacturer's advertising strategy, $V_{R}^{D}-V_{R}^{N}$ $>0 \forall \phi \in(0,3-\sqrt{5})$

(b) When goodwill does not depend on the retailer's advertising strategy, $V_{R}^{D}-V_{R}^{N}$ $>0 \forall \phi \in(0,0.9249)$

(c) When goodwill depends on both players' advertising strategies, $V_{R}^{D}-V_{R}^{N}$ $>0 \forall \phi \in(0,3-\sqrt{5})$ and $\forall \phi \in(3-\sqrt{5}, 0.9249) \Leftrightarrow b>a$.

Further, when $\phi=3-\sqrt{5}, V_{i}^{D}=V_{i}^{N}$. Complementing a RSC with a support program lessens negative effect of contracting. Although complementing a RSC with a support program lowers the retailer's myopia, the combination of coordination mechanisms not longer leads to a Pareto improving situation when the sharing parameter is too high. Another condition needed is that the retailer advertising effectiveness should be substantially higher than the manufacturer's one. This is quite convincible as the support program has the purpose of increasing retailer advertising efforts, which turn out to decrease profits for low value of retailer advertising effectiveness. When the retailer has enough bargaining power to set up a proper sharing parameter value, complementing a RSC with a support program leads to a payoff-Pareto-improving situation.

Corollary 4 Assume that $\rho=\delta$, when the players use the same advertising media $(a=b)$ it results that $V_{M}^{D}-V_{M}^{N}>0, \forall \phi(0,1)$ and $V_{R}^{D}-V_{R}^{N}>0, \forall \phi(0,0.8572)$. Thus, the implementation of a RSC complemented with a support program makes the manufacturer always better off, while the retailer's economic advantages depend on the sharing parameter values. Equal players' advertising effectiveness enlarges the sharing parameter range inside which both players are economically better off and thus increases the chances to coordinate a marketing channel when a RSC and a support program are simultaneously implemented.

\section{Conclusions}

Contrary to the literature on operations that lauds the adoption of a RSC to mitigate the double marginalization effect and increase profits, we show that this contract scheme is payoffPareto-improving only in a few cases. This is due to our atypical assumption for which the player who transfers a part of his revenues does also advertising. Under this assumption a RSC misaligns players' motivations to invest in advertising and destroys economic value. 
Table 1 Summary of the findings

The goodwill does not depend on the manufacturer's media
Manufacturer and retailer The goodwill does not use the same advertising depend on the retailer's media media

\begin{tabular}{|c|c|c|c|}
\hline $\begin{array}{l}\text { No support strategy } \\
(B=0)\end{array}$ & $\begin{array}{l}V_{M}^{C}-V_{M}^{N}>0, \forall \phi(0,1) \\
V_{R}^{C}-V_{R}^{N}>0 \\
\forall \phi(0,3-\sqrt{5})\end{array}$ & $\begin{array}{l}V_{R}^{C}-V_{R}^{N}>0, \\
\forall \phi(0,0.6377)\end{array}$ & $\begin{array}{l}V_{M}^{C}-V_{M}^{N}>0, \forall \phi(0,3-\sqrt{5}) \\
V_{R}^{C}-V_{R}^{N}>0, \forall \phi(0,1)\end{array}$ \\
\hline $\begin{array}{l}\text { Support strategy } \\
(B>0)\end{array}$ & $\begin{array}{l}V_{M}^{D}-V_{M}^{N}>0, \forall \phi(0,1) \\
V_{R}^{D}-V_{R}^{N}>0 \\
\forall \phi(0,0.9249)\end{array}$ & $\begin{array}{l}V_{R}^{D}-V_{R}^{N}>0, \\
\forall \phi(0,0.8572)\end{array}$ & $V_{R}^{D}-V_{R}^{N}>0, \forall \phi(0,3-\sqrt{5})$ \\
\hline $\begin{array}{l}\text { Support strategy } \\
(B>0)\end{array}$ & $\begin{array}{l}V_{M}^{D}-V_{M}^{C}>0, \forall \phi(0,1) \\
V_{R}^{D}-V_{R}^{C}>0, \forall \phi(0,1)\end{array}$ & & \\
\hline
\end{tabular}

Offering a support program represents thus a valid alternative to alleviate the drawbacks a RSC creates, again aligning players' motivations to focus on dynamic tools. Even though, setting up high sharing parameter values lowers the benefits that a support program implies and a RSC continues to lessen players' payoffs. In our research, we purposely disregard the case when a WPC is complemented with a support program because we get results aligned to the literature of marketing (e.g., Jørgensen et al. 2003) as well as the case of full support program because it leads to a centralized scenario in which the retailer transfers all her revenues to the manufacturer. Finally, a support program is generally payoff-Pareto-improving while the adoption of a RSC rarely leads to the same result. Table 1 summarizes the findings of this research.

Future research could be developed in several directions. We could evaluate the simultaneous use of support programs and other contracts (e.g., flexible quantity), thereby integrating coordination mechanisms generally used in marketing and operations management. Furthermore, marketing and operational tools, such as quality, promotion, and new product development, could be used to evaluate the effectiveness of a RSC. Other contracts (e.g. a reverse RSC) could be evaluated for their ability to achieve coordination in marketing channels. Finally, case studies and empirical research are needed in this domain, where the use of a two-parameter contract seems to be the focus in only a few business applications (e.g., the video rental industry).

Open Access This article is distributed under the terms of the Creative Commons Attribution License which permits any use, distribution, and reproduction in any medium, provided the original author(s) and the source are credited.

\section{Appendix}

Proof of Proposition 1 We need to establish the existence of bounded and continuously differentiable value functions $V_{M}^{N}(G), V_{R}^{N}(G)$ such that there exists a unique solution $G^{N}(t)$ to (1) and the HJB equations. The players' HJBs in the non-coordinated scenario are: 


$$
\begin{aligned}
& \rho V_{M}^{N}=\left(\theta \sqrt{G^{N}}-\beta p^{N}\right) \omega^{N}-\frac{A_{M}^{N 2}}{2}+V_{M}^{N^{\prime}}\left(a A_{M}^{N}+b A_{R}^{N}-\delta G^{N}\right), \\
& \rho V_{R}^{N}=\left(\theta \sqrt{G^{N}}-\beta p^{N}\right)\left(p^{N}-\omega^{N}\right)-\frac{A_{R}^{N 2}}{2}+V_{R}^{N^{\prime}}\left(a A_{M}^{N}+b A_{R}^{N}-\delta G^{N}\right)
\end{aligned}
$$

Since the game is played à la Stackelberg and the manufacturer is the leader, we first determine the necessary conditions of the retailer as:

$$
\begin{aligned}
& p^{N}=\frac{\theta \sqrt{G^{N}}+\beta \omega^{N}}{2 \beta}, \\
& A_{R}^{N}=b V_{R}^{N}
\end{aligned}
$$

Substituting (32) and (33) into the manufacturer's HJB equation, we obtain:

$$
\rho V_{M}^{N}=\left(\frac{\theta \sqrt{G^{N}}-\beta \omega^{N}}{2}\right) \omega^{N}-\frac{A_{M}^{N 2}}{2}+V_{M}^{N^{\prime}}\left(a A_{M}^{N}+b^{2} V_{R}^{N}-\delta G\right)
$$

Thus, we compute the manufacturer's necessary conditions given by

$$
\begin{aligned}
& \omega^{N}=\frac{\theta \sqrt{G^{N}}}{2 \beta}, \\
& A_{M}^{N}=a V_{M}^{N}
\end{aligned}
$$

Finally, substituting the wholesale price into the retailer price, we obtain:

$$
p^{N}=\frac{3 \theta \sqrt{G^{N}}}{4 \beta}
$$

While substituting (33), (35), (36) and (37) into (31) and (34), the HJBs turn out to be:

$$
\begin{aligned}
& 8 \beta \rho V_{M}^{N}=\theta^{2} G^{N}+4 \beta V_{M}^{N^{\prime}}\left(a^{2} V_{M}^{N^{\prime}}+2 b^{2} V_{R}^{N^{\prime}}-2 \delta G^{N}\right), \\
& 16 \beta \rho V_{R}^{N}=\theta^{2} G^{N}+8 \beta V_{R}^{N^{\prime}}\left(2 a^{2} V_{M}^{N^{\prime}}+b^{2} V_{R}^{N^{\prime}}-2 \delta G^{N}\right)
\end{aligned}
$$

We conjecture linear value functions, $V_{M}^{N}=\varphi_{1}^{N} G^{N}+\varphi_{2}^{N}, V_{M}^{N^{\prime}}=\varphi_{1}^{N^{\prime}}$, and $V_{R}^{N}=\varsigma_{1}^{N} G^{N}+$ $\varsigma_{2}^{N}, V_{R}^{N^{\prime}}=\varsigma_{1}^{N^{\prime}}$ where $\varphi_{1}^{N}, \varphi_{2}^{N}, \varsigma_{1}^{N}$ and $\varsigma_{2}^{N}$ are the constant parameters to be identified. Substituting $V_{M}^{N}(G)$ and $V_{R}^{N}(G)$ and their derivatives in (38) and (39), we obtain:

$$
\begin{aligned}
& 8 \beta \rho\left(\varphi_{1}^{N} G^{N}+\varphi_{2}^{N}\right)=\theta^{2} G^{N}+4 \beta \varphi_{1}^{N^{\prime}}\left(a^{2} \varphi_{1}^{N^{\prime}}+2 b^{2} \varsigma_{1}^{N^{\prime}}-2 \delta G^{N}\right), \\
& 16 \beta \rho\left(\varsigma_{1}^{N} G^{N}+\varsigma_{2}^{N}\right)=\theta^{2} G^{N}+8 \beta \varsigma_{1}^{N^{\prime}}\left(2 a^{2} \varphi_{1}^{N^{\prime}}+b^{2} \varsigma_{1}^{N^{\prime}}-2 \delta G^{N}\right)
\end{aligned}
$$

where the value parameters may be easily identified as follows:

$$
\begin{aligned}
\varphi_{1}^{N} & =\frac{\theta^{2}}{8 \beta(\rho+\delta)}, \\
\varphi_{2}^{N} & =\frac{\varphi_{1}^{N}\left(a^{2} \varphi_{1}^{N}+2 b^{2} \varsigma_{1}^{N}\right)}{2 \rho},
\end{aligned}
$$




$$
\begin{aligned}
\varsigma_{1}^{N} & =\frac{\theta^{2}}{16 \beta(\rho+\delta)}, \\
\varsigma_{2}^{N} & =\frac{\varsigma_{1}^{N}\left(2 a^{2} \varphi_{1}^{N}+b^{2} \varsigma_{1}^{N}\right)}{2 \rho}
\end{aligned}
$$

Proof of Proposition 2 We need to establish the existence of bounded and continuously differentiable value functions $V_{M}^{C}(G), V_{R}^{C}(G)$ such that there exists a unique solution $G^{C}(t)$ to (1) and the HJB equations. The players' HJBs in the coordinated scenario are:

$$
\begin{aligned}
& \rho V_{M}^{C}=\left(\theta \sqrt{G^{C}}-\beta p^{C}\right)\left(\omega^{C}+p^{C} \phi\right)-\frac{A_{M}^{C 2}}{2}+V_{M}^{C^{\prime}}\left(a A_{M}^{C}+b A_{R}^{C}-\delta G^{C}\right), \\
& \rho V_{R}^{C}=\left(\theta \sqrt{G^{C}}-\beta p^{C}\right)\left(p^{C}(1-\phi)-\omega^{C}\right)-\frac{A_{R}^{C 2}}{2}+V_{R}^{C^{\prime}}\left(a A_{M}^{C}+b A_{R}^{C}-\delta G^{C}\right)
\end{aligned}
$$

Since the coordinated scenario is also played $\grave{a}$ la Stackelberg and the manufacturer is the leader, we first determine the necessary conditions of the retailer, as given by:

$$
\begin{aligned}
& p^{C}=\frac{\beta \omega^{C}+\theta \sqrt{G^{C}}(1-\phi)}{2 \beta(1-\phi)}, \\
& A_{R}^{C}=b V_{R}^{C^{\prime}}
\end{aligned}
$$

Substituting (48) and (49) into the manufacturer's HJB equation, we obtain:

$$
\begin{aligned}
\rho V_{M}^{C}= & \left(\frac{\theta \sqrt{G^{C}}(1-\phi)-\beta \omega^{C}}{2(1-\phi)}\right)\left(\frac{\beta(2-\phi) \omega^{C}+\theta \sqrt{G^{C}}(1-\phi) \phi}{2 \beta(1-\phi)}\right)-\frac{A_{M}^{C 2}}{2} \\
& +V_{M}^{C^{\prime}}\left(a A_{M}^{C}+b A_{R}^{C}-\delta G^{C}\right)
\end{aligned}
$$

Thus, we compute the manufacturer's necessary conditions given as follows:

$$
\begin{aligned}
& \omega^{C}=\frac{\theta \sqrt{G^{C}}(1-\phi)^{2}}{\beta(2-\phi)}, \\
& A_{M}^{C}=a V_{M}^{C^{\prime}}
\end{aligned}
$$

Finally, substituting the wholesale price into the retailer price, we obtain:

$$
p^{C}=\frac{\theta \sqrt{G^{C}}(3-2 \phi)}{2 \beta(2-\phi)}
$$

While substituting (49), (51), (52) and (53) into (47) and (50), the HJBs turn out to be:

$$
\begin{aligned}
& 4 \beta(2-\phi) \rho V_{M}^{C}=\theta^{2} G^{C}+2 \beta(2-\phi) V_{M}^{C^{\prime}}\left(a^{2} V_{M}^{C^{\prime}}+2 b^{2} V_{R}^{C^{\prime}}-2 \delta G^{C}\right), \\
& 4 \beta(2-\phi)^{2} \rho V_{R}^{C}=\theta^{2} G^{C}(1-\phi)+2 \beta(2-\phi)^{2} V_{R}^{C^{\prime}}\left(2 a^{2} V_{M}^{C^{\prime}}+b^{2} V_{R}^{C^{\prime}}-2 \delta G^{C}\right)
\end{aligned}
$$

Linear value functions satisfy (54) and (55); therefore, we conjecture $V_{M}^{C}=\varphi_{1}^{C} G^{C}+\varphi_{2}^{C}$ and $V_{R}^{C}=\varsigma_{1}^{C} G^{C}+\varsigma_{2}^{C}$, where $\varphi_{1}^{C}, \varphi_{2}^{C}, \varsigma_{1}^{C}$ and $\varsigma_{2}^{C}$ are the constant parameters to be identified. 
Substituting $V_{M}^{C}(G)$ and $V_{R}^{C}(G)$ and their derivatives into (54) and (55), we obtain

$$
\begin{aligned}
& 4 \beta(2-\phi) \rho\left(\varphi_{1}^{C} G^{C}+\varphi_{2}^{C}\right)=\theta^{2} G^{C}+2 \beta(2-\phi) \varphi_{1}^{C}\left(a^{2} \varphi_{1}^{C}+2 b^{2} \varsigma_{1}^{C}-2 \delta G^{C}\right), \\
& 4 \beta(2-\phi)^{2} \rho\left(\varsigma_{1}^{C} G^{C}+\varsigma_{2}^{C}\right) \\
& \quad=\theta^{2} G^{C}(1-\phi)+2 \beta(2-\phi)^{2} \varsigma_{1}^{C}\left(2 a^{2} \varphi_{1}^{C}+b^{2} \varsigma_{1}^{C}-2 \delta G^{C}\right)
\end{aligned}
$$

where the value parameter may be easily identified as follows:

$$
\begin{aligned}
& \varphi_{1}^{C}=\frac{\theta^{2}}{4 \beta(2-\phi)(\rho+\delta)}, \\
& \varphi_{2}^{C}=\frac{\varphi_{1}^{C}\left(a^{2} \varphi_{1}^{C}+2 b^{2} \varsigma_{1}^{C}\right)}{2 \rho}, \\
& \varsigma_{1}^{C}=\frac{\theta^{2}(1-\phi)}{4 \beta(2-\phi)^{2}(\rho+\delta)}, \\
& \varsigma_{2}^{C}=\frac{\varsigma_{1}^{C}\left(2 a^{2} \varphi_{1}^{C}+b^{2} \varsigma_{1}^{C}\right)}{2 \rho}
\end{aligned}
$$

Proof of Proposition 10 We need to establish the existence of bounded and continuously differentiable value functions $V_{M}^{D}(G), V_{R}^{D}(G)$ such that there exists a unique solution $G^{D}(t)$ to (1) and the HJB equations. The players' HJBs in this scenario are:

$$
\begin{aligned}
\rho V_{M}^{D}= & \left(\theta \sqrt{G^{D}}-\beta p^{D}\right)\left(\omega^{D}+p^{D} \phi\right)-\frac{A_{R}^{D 2} B}{2}-\frac{A_{M}^{D 2}}{2} \\
& +V_{M}^{D^{\prime}}\left(a A_{M}^{D}+b A_{R}^{D}-\delta G^{D}\right), \\
\rho V_{R}^{D}= & \left(\theta \sqrt{G^{D}}-\beta p^{D}\right)\left(p^{D}(1-\phi)-\omega^{D}\right)-\frac{A_{R}^{D 2}(1-B)}{2} \\
& +V_{R}^{D^{\prime}}\left(a A_{M}^{D}+b A_{R}^{D}-\delta G^{D}\right)
\end{aligned}
$$

Also this scenario is played à la Stackelberg and the manufacturer is the leader, thus we determine the retailer's necessary conditions, who decides the retail price and advertising efforts, as given by:

$$
\begin{aligned}
p^{D} & =\frac{\theta \sqrt{G^{D}}(1-\phi)+\beta \omega^{D}}{2 \beta(1-\phi)}, \\
A_{R}^{D} & =\frac{b V_{R}^{D^{\prime}}}{1-B}
\end{aligned}
$$


Substituting (64) and (65) into the manufacturer's HJB equation, we obtain:

$$
\begin{aligned}
\rho V_{M}^{D}= & \left(\frac{\theta \sqrt{G^{D}}(1-\phi)-\beta \omega^{D}}{2(1-\phi)}\right)\left(\frac{\omega^{D} \beta(2-\phi)+\theta \sqrt{G^{D}}(1-\phi) \phi}{2 \beta(1-\phi)}\right)-\left(\frac{b V_{R}^{D^{\prime}}}{1-B}\right)^{2} \frac{B}{2} \\
& -\frac{A_{M}^{D 2}}{2}+V_{M}^{D^{\prime}}\left(a A_{M}^{D}+\frac{b^{2} V_{R}^{D^{\prime}}}{1-B}-\delta G^{D}\right)
\end{aligned}
$$

Thus, we compute the manufacturer's necessary conditions given by the wholesale price, advertising efforts, and participation rate as follows:

$$
\begin{aligned}
& \omega^{D}=\frac{\theta \sqrt{G^{D}}(1-\phi)^{2}}{\beta(2-\phi)}, \\
& A_{M}^{D}=a V_{M}^{D^{\prime}}, \\
& B=\frac{2 V_{M}^{D^{\prime}}-V_{R}^{D^{\prime}}}{2 V_{M}^{D^{\prime}}+V_{R}^{D^{\prime}}}
\end{aligned}
$$

Finally, substituting the wholesale price into the retailer price, we obtain:

$$
p^{D}=\frac{\theta \sqrt{G^{D}}(3-2 \phi)}{2 \beta(2-\phi)}
$$

Substitute (65), (67), (68), (69) and (70) into the HJBs and assume linear $V_{M}^{D}=\varphi_{1}^{D} G^{D}+\varphi_{2}^{D}$ and $V_{R}^{D}=\varsigma_{1}^{D} G^{D}+\varsigma_{2}^{D}$. Following the same procedure as in the proof of Propositions 1 and 2 provides the remaining results of Proposition 10.

\section{References}

Chintagunta, P. K., \& Jain, D. (1992). A dynamic model of channel member strategies for marketing expenditures. Marketing Science, 11(2), 117-134.

Cachon, G. P. (2003). Supply chain coordination with contracts. In S. Graves \& T. de Kok (Eds.), Handbooks in operations research and management science: supply chain management. Amsterdam: NorthHolland.

Cachon, G. P., \& Lariviere, M. A. (2005). Supply chain coordination with revenues sharing contracts: strength and limitations. Management Science, 51(1), 30-44.

Dana, J. D. Jr., \& Spier, K. E. (2001). Revenue sharing and vertical control in the video rental industry. The Journal of Industrial Economics, 49(3), 223-245.

De Giovanni, P. (2011a). Quality improvement vs. advertising support: which strategy works better for a manufacturer. European Journal of Operations Research, 208(2), 119-130.

De Giovanni, P. (2011b). Environmental collaboration through a reverse revenue sharing contract. Annals of Operations Research, 6, 1-23.

El Ouardighi, F., \& Kim, B. (2010). Supply quality management with wholesale price and revenue-sharing contracts under horizontal competition. European Journal of Operational Research, 206(2), 329-340.

El Ouardighi, F., Jørgensen, S., \& Pasin, F. (2008). A dynamic game of operations and marketing management in a supply chain. International Game Theory Review, 34, 59-77.

Fleischmann, M., van Nunen, J., \& Grave, B. (2002). Integrating closed-loop supply chains and spare parts in IBM.

Geng, Q., \& Mallik, S. (2007). Inventory competition and allocation in a multi-channel distribution system. European Journal of Operational Research, 182(2), 704-729.

He, X., Prasad, A., \& Sethi, S. (2009). Cooperative advertising and pricing in a dynamic stochastic supply chain: feedback Stackelberg strategies. Production and Operations Management, 18, 78-94.

Jeuland, A. P., \& Shugan, S. M. (1983). Managing channel profits. Marketing Science, 2, 239-272. 
Jørgensen, S., \& Zaccour, G. (2003a). Channel coordination over time: incentive equilibria and credibility. Journal of Economic Dynamics \& Control, 27, 801-822.

Jørgensen, S., \& Zaccour, G. (2003b). A differential game of retailer promotions. Automatica, 39, 11451155.

Jørgensen, S., \& Zaccour, G. (2005). Differential games in marketing. New York: Springer.

Jørgensen, S., Sigué, S. P., \& Zaccour, G. (2000). Dynamic cooperative advertising in a channel. Journal of Retailing, 76, 71-92.

Jørgensen, S., Sigué, S. P., \& Zaccour, G. (2001). Stackelberg leadership in a marketing channel. International Game Theory Review, 3, 13-26.

Jørgensen, S., Taboubi, S., \& Zaccour, G. (2003). Retail promotions with negative brand image effects: is cooperation possible? European Journal of Operational Research, 150, 395-405.

Jørgensen, S., Taboubi, S., \& Zaccour, G. (2006). Incentives for retailer promotion in a marketing channel. Annals of the International Society of Dynamic Games, 8, 365-378.

Kadlec, D., Keith, A., \& Aixa, M. P. (1998). How blockbuster changed the rules. Time Magazine, August, 03.

Karray, S., \& Zaccour, G. (2006). Could co-op advertising be a manufacturer's counterstrategy to store brands? Journal of Business Research, 59, 1008-1015.

Moorthy, K. S. (1988). Managing channel profits: comment. Marketing Science, 6, 375-379.

Nerlove, M., \& Arrow, K. J. (1962). Optimal advertising policy under dynamic conditions. Economica, 39(114), 129-142.

Pan, K., Lai, K. K., Leung, S. C. H., \& Xiao, D. (2010). Revenue-sharing versus wholesale price mechanisms under different channel power structures. European Journal of Operational Research, 203, 532-538.

Pasternack, B. (2002). Using revenue sharing to achieve channel coordination for a newsboy type inventory model. In J. Geunes, P. Pardalos, \& H. E. Romeijn (Eds.), Supply chain management: models, applications and research. Dordrecht: Kluwer Academic.

Taboubi, S., \& Zaccour, G. (2002). Impact of Retailer's myopia on channel's strategies. In Optimal control and differential games. Essays in honour of Steffen Jorgensen. Dordrecht: Kluwer Academic.

Tirole, J. (1988). The theory of industrial organization. Cambridge: MIT Press.

Tsay, A. A., Nahmias, S., \& Agrawal, N. (1999). Modeling in supply chain contract. In S. Tayur, R. Ganeshan, \& M. Magazine (Eds.), Quantitative models for supply chain management. Boston: Kluwer Academic.

Wang, Y. (2006). Joint pricing-production decisions in supply chains of complementary products with uncertain demand. Operations Research, 54, 1110-1127.

Wong, W. K., Qi, J., \& Leung, S. Y. S. (2009). Coordinating supply chains with sales rebate contracts and vendor-management inventory. International Journal of Production Economics, 1(120), 151-161.

Yao, Z., Leung, S. C. H., \& Lai, K. K. (2008). Manufacturer's revenue-sharing contract and retail competition. European Journal of Operational Research, 186, 637-651. 